\title{
Species Over a Finite Field
}

\author{
ANTHONY HENDERSON \\ School of Mathematics and Statistics, University of Sydney, NSW 2006, Australia
}

anthonyh@maths.usyd.edu.au

Received May 22, 2003; Accepted March 10, 2004

\begin{abstract}
We generalize Joyal's theory of species to the case of functors from the groupoid of finite sets to the category of varieties over $\mathbb{F}_{q}$. These have cycle index series defined by counting fixed points of twisted Frobenius maps. We give an application to configuration spaces.
\end{abstract}

Keywords: species, finite field, configuration space

\section{Introduction}

In [4], Joyal defined a species (of structures) to be a functor $A: \mathbf{B} \rightarrow \mathbf{B}$, where $\mathbf{B}$ is the groupoid of finite sets and bijections between them. One should think of $A$ as a "kind" of structure on a finite set, and of $A(I)$ as the set of structures of that kind on the set $I$. For instance, $A$ could be the species $L$ of total orders; it is clear how a bijection $I \stackrel{\sim}{\rightarrow} I^{\prime}$ defines a bijection between the set $L(I)$ of total orders on $I$ and the set $L\left(I^{\prime}\right)$ of total orders on $I^{\prime}$.

As well as providing a useful language in which to discuss many combinatorial constructions, species are of great use in certain enumerative problems, particularly those involving labelled trees. Their utility comes about via the definition of the cycle index series $Z_{A}$ of a species $A$, which is an element of the formal power series ring $\mathbb{Q}\left[\left[x_{1}, x_{2}, \ldots\right]\right]$, encoding the number of fixed points on $A(I)$ of the permutation $A(w)$ for any permutation $w$ of $I$. (See Section 2 for the definition.) Joyal showed that many natural operations on species correspond to natural operations on their cycle index series: most significantly, substitution of species corresponds to plethysm of series (see Theorem 2.1).

Variants of the notion of species abound. In Section 3, for later use, we will discuss the notion of $\Gamma$-species, which is a functor from $\mathbf{B}$ to the category of sets with a $\Gamma$-action, for some finite group $\Gamma$. We will also give in full a proof of the generalization of Joyal's result to this context (see Theorem 3.1).

The linear analogue of a species, a tensor species or $\mathbb{S}$-module, is a functor from $\mathbf{B}$ to the category of vector spaces over a fixed base field. Examples of $\mathbb{S}$-modules are the operads encountered in quantum cohomology theory (see [9, Chapter IV], for instance). These too have attached power series, which this time encode traces rather than numbers of fixed points. (They are usually called characteristics and disguised as symmetric functions-see Section 4.) Again, Joyal observed that substitution of $\mathbb{S}$-modules corresponds to plethysm of characteristics (see Theorem 4.1). 
Further variants involve functors from $\mathbf{B}$ to some geometric or topological category (for instance, topological operads). These are generally studied via their cohomology, which is a graded $\mathbb{S}$-module: after all, if the fixed-point sets are infinite, the cycle index series cannot be defined in the usual way. However, the point of this paper is to indicate a case when cohomology is not required, namely the case of species over $\mathbb{F}_{q}$, where $\mathbb{F}_{q}$ is a finite field. These are functors $X$ from $\mathbf{B}$ to the category of varieties over $\mathbb{F}_{q}$, and they have cycle index series which encode the number of fixed points on $X(I)$ of the twisted Frobenius map $X(w) F$, for any permutation $w$ of $I$. The main result of this paper (Theorem 5.1) is that substitution corresponds to plethysm in this context.

One would expect species over finite fields to be of most use in counting the Frobenius fixed points of varieties which have a stratification with strata parametrized by some sort of labelled trees. In Section 6, we will illustrate the use of Theorem 5.1 in the case of configuration spaces and their Fulton-MacPherson compactifications. As will be explained, this application could also be handled via the cohomological method (in this case, using the Grothendieck Trace Formula in $l$-adic cohomology). Moreover, the analogous arguments for complex varieties and singular cohomology can be found in an unpublished paper of Getzler ([3]), so the results of Section 6 are not original in essence. But it seemed worthwhile to present them, since the formalism of species over $\mathbb{F}_{q}$ is cleaner and more elementary than the cohomological approach.

\section{Species of structures}

As mentioned in the introduction, a species is a functor $A: \mathbf{B} \rightarrow \mathbf{B}$, where $\mathbf{B}$ is the groupoid of finite sets and bijections between them. (Of course, one could equally well say it is a functor from $\mathbf{B}$ to the category of finite sets, since the image would automatically lie in $\mathbf{B}$.) The definition of isomorphism of species is the usual one for functors. In addition to [4], a good reference for the theory of species is [1].

For any nonnegative integer $n$, write $[n]$ for $\{1,2, \ldots, n\}$, and $S_{n}$ for its group of permutations. For a species $A$, write $A[n]$ for $A([n])$; by definition, this is a finite set with an $S_{n}$-action. For $w \in S_{n}$, we will write the induced permutation on $A[n]$ simply as $w$, not as $A(w)$. Generally we are interested in the numbers of fixed points $\left|A[n]^{w}\right|$ for various permutations $w \in S_{n}$. These numbers are collected for convenience in the $c y$ cle index series of $A$, which is a formal power series in the variables $x_{1}, x_{2}, \ldots$, defined by

$$
Z_{A}\left(x_{1}, x_{2}, \ldots\right):=\sum_{n \geq 0} \frac{1}{n !} \sum_{w \in S_{n}}\left|A[n]^{w}\right| x_{w} \in \mathbb{Q}\left[\left[x_{1}, x_{2}, \ldots\right]\right]
$$

where if $w$ has cycle-type $\left(i^{m_{i}}\right), x_{w}:=x_{1}^{m_{1}} x_{2}^{m_{2}} \ldots$ Note that $\left|A[n]^{w}\right|$ depends only on the cycle-type (conjugacy class) of $w$, so $Z_{A}$ can be rewritten (using the partition notation of [7])

$$
Z_{A}=\sum_{n \geq 0} \sum_{\lambda \vdash n} z_{\lambda}^{-1}\left|A[n]^{w_{\lambda}}\right| x_{1}^{m_{1}(\lambda)} x_{2}^{m_{2}(\lambda)} \ldots
$$


where $w_{\lambda}$ denotes an arbitrary element of cycle-type $\lambda$. In particular, all the numbers $\left|A[n]^{w}\right|$ are determined by $Z_{A}$.

There are also two significant specializations of $Z_{A}$ :

$$
A(x):=Z_{A}(x, 0,0, \ldots)=\sum_{n \geq 0} \frac{|A[n]|}{n !} x^{n},
$$

the exponential generating series of $A$, and

$$
\tilde{A}(x):=Z_{A}\left(x, x^{2}, x^{3}, \ldots\right)=\sum_{n \geq 0}\left|S_{n} \backslash A[n]\right| x^{n},
$$

the type generating series of $A$. To get the last equality, we have used the following special case of the Lemma formerly known as Burnside's:

$$
\left|S_{n} \backslash A[n]\right|=\frac{1}{n !} \sum_{w \in S_{n}}\left|A[n]^{w}\right| .
$$

If $A$ and $B$ are two species, there are species $A+B$ and $A \cdot B$, defined on a finite set $I$ by

$$
\begin{aligned}
(A+B)(I) & =A(I) \amalg B(I), \\
(A \cdot B)(I) & =\coprod_{I=I_{1} \amalg I_{2}} A\left(I_{1}\right) \times B\left(I_{2}\right) .
\end{aligned}
$$

The definitions of these functors on bijections $I \stackrel{\sim}{\rightarrow} I^{\prime}$ are obvious. It is easy to see that

$$
Z_{A+B}=Z_{A}+Z_{B}, Z_{A \cdot B}=Z_{A} Z_{B} .
$$

There is also a basic notion of substitution of species, which associates to two species $A$ and $B$, where $B(\emptyset)=\emptyset$, a third species $A \circ B$. (This is not the composition of the functors $A$ and $B$, although it does correpond to composition of the associated analytic functors-see [5].) For a finite set $I$, one defines

$$
(A \circ B)(I):=\coprod_{\pi \in \operatorname{Par}(I)}\left(A(\pi) \times \prod_{J \in \pi} B(J)\right),
$$

where $\operatorname{Par}(I)$ is the set of partitions of $I$, i.e. sets of non-empty disjoint subsets whose union equals $I$. The definition of $A \circ B$ on bijections $I \stackrel{\sim}{\rightarrow} I^{\prime}$ is obvious. It is easy to see that $\circ$ is associative up to isomorphism: more precisely, it gives a monoidal structure on the category of species $A$ which satisfy $A(\emptyset)=\emptyset$. The species of singletons $E_{1}$, defined by

$$
E_{1}(I)= \begin{cases}\{I\}, & \text { if }|I|=1 \\ \emptyset, & \text { otherwise }\end{cases}
$$

is an identity for this monoidal structure. 
The corresponding operation on cycle index series is plethysm: if $f, g \in \mathbb{Q}\left[\left[x_{1}, x_{2}, \ldots\right]\right]$, and $g$ has zero constant term, define

$$
(f \circ g)\left(x_{1}, x_{2}, \ldots\right):=f\left(g\left(x_{1}, x_{2}, \ldots\right), g\left(x_{2}, x_{4}, \ldots\right), g\left(x_{3}, x_{6}, \ldots\right), \ldots\right) .
$$

This too is an associative operation, with identity $x_{1}\left(=Z_{E_{1}}\right)$.

Theorem 2.1 (Joyal) If $A$ and $B$ are species and $B(\emptyset)=\emptyset$, then

$$
Z_{A \circ B}=Z_{A} \circ Z_{B}
$$

For a proof, see [4, Proposition 14] or [1, Section 4.3], or take the special case $\Gamma=\{1\}$ of the proof of Theorem 3.1 below. See [1] for a plethora of applications of Theorem 2.1 to enumerative combinatorics.

\section{3. $\Gamma$-species}

In this section we examine one of many possible extensions of the definition of species. If $\Gamma$ is a finite group, define a $\Gamma$-species to be a functor from $\mathbf{B}$ to the category of finite sets with a $\Gamma$-action, where the morphisms are $\Gamma$-equivariant maps. (The previous definition of species is recovered as the case $\Gamma=\{1\}$.) Let $\mathbb{Q}\left[\left[x_{1}, x_{2}, \ldots\right]\right]_{\Gamma}$ be the set of functions from $\Gamma$ to $\mathbb{Q}\left[\left[x_{1}, x_{2}, \ldots\right]\right]$. If $A$ is a $\Gamma$-species, then $A[n]$ is a finite set with commuting actions of $S_{n}$ and $\Gamma$, and we define the cycle index series $Z_{A} \in \mathbb{Q}\left[\left[x_{1}, x_{2}, \ldots\right]\right]_{\Gamma}$ by

$$
Z_{A}(\gamma):=\sum_{n \geq 0} \frac{1}{n !} \sum_{w \in S_{n}}\left|A[n]^{w \gamma}\right| x_{w},
$$

where $A[n]^{w \gamma}$ is the set of fixed points of the composition $w \gamma$.

Note that $Z_{A}(1)$ is the cycle index series of $A$ considered as an ordinary species (forgetting the $\Gamma$-actions). We also have specializations of $Z_{A}$ analogous to (2.2) and (2.3):

$$
\begin{aligned}
Z_{A}(\gamma)(x, 0,0, \ldots) & =\sum_{n \geq 0} \frac{\left|A[n]^{\gamma}\right|}{n !} x^{n}, \\
Z_{A}(\gamma)\left(x, x^{2}, x^{3}, \ldots\right) & =\sum_{n \geq 0}\left|\left(S_{n} \backslash A[n]\right)^{\gamma}\right| x^{n} .
\end{aligned}
$$

The second equality comes from the following easy variant of (2.4):

$$
\left|\left(S_{n} \backslash A[n]\right)^{\gamma}\right|=\frac{1}{n !} \sum_{w \in S_{n}}\left|A[n]^{w \gamma}\right| .
$$

The definitions of addition, multiplication, and substitution given in the previous section all work identically for $\Gamma$-species. It is also clear that the first two still correspond to 
addition and multiplication of cycle index series, using the pointwise ring structure on $\mathbb{Q}\left[\left[x_{1}, x_{2}, \ldots\right]\right]_{\Gamma}$. We define the plethysm of $f, g \in \mathbb{Q}\left[\left[x_{1}, x_{2}, \ldots\right]\right]_{\Gamma}$, where $g(\gamma)$ has zero constant term for all $\gamma$, by

$$
(f \circ g)(\gamma):=f(\gamma)\left(g(\gamma)\left(x_{1}, x_{2}, \ldots\right), g\left(\gamma^{2}\right)\left(x_{2}, x_{4}, \ldots\right), \ldots\right) .
$$

With this definition, the obvious generalization of Theorem 2.1 holds:

Theorem 3.1 If $A$ and $B$ are $\Gamma$-species and $B(\emptyset)=\emptyset$, then

$$
Z_{A \circ B}=Z_{A} \circ Z_{B} .
$$

Proof: By definition,

$$
Z_{A \circ B}(\gamma)=\sum_{n \geq 0} \frac{1}{n !} \sum_{w \in S_{n}}\left|\left(\coprod_{\pi \in \operatorname{Par}[n]}\left(A(\pi) \times \prod_{J \in \pi} B(J)\right)\right)^{w \gamma}\right| x_{w} .
$$

It is clear that only those partitions in $\operatorname{Par}[n]$ which are invariant under $w$ contribute to the count of $w \gamma$-fixed points. Hence

$$
Z_{A \circ B}(\gamma)=\sum_{n \geq 0} \frac{1}{n !} \sum_{\pi \in \operatorname{Par}[n]} \sum_{\substack{w \in S_{n} \\ w, \pi=\pi}}\left|\left(A(\pi) \times \prod_{J \in \pi} B(J)\right)^{w \gamma}\right| x_{w} .
$$

For any $m \geq 0$ and $n_{1}, n_{2}, \ldots, n_{m} \geq 1$, define

$$
I^{n_{1}, \ldots, n_{m}}=\left\{(j, k) \mid j \in[m], k \in\left[n_{j}\right]\right\} .
$$

Let $\pi^{n_{1}, \ldots, n_{m}}$ be the obvious partition $\left\{\pi_{j}^{n_{1}, \ldots, n_{m}} \mid j \in[m]\right\}$ of $I^{n_{1}, \ldots, n_{m}}$, where $\pi_{j}^{n_{1}, \ldots, n_{m}}=$ $\left\{(j, k) \mid k \in\left[n_{j}\right]\right\}$, and let $S_{n_{1}, \ldots, n_{m}}$ be the group of permutations of $I^{n_{1}, \ldots, n_{m}}$ which fix this partition (not necessarily fixing each part individually). If $\pi \in \operatorname{Par}[n]$ has $m$ parts, we can number these parts $\pi_{1}, \ldots, \pi_{m}$ in $m$ ! ways. Letting $n_{1}, \ldots, n_{m}$ be their sizes, there are then $\frac{n !}{n_{1} ! \ldots n_{m} !}$ bijections between $[n]$ and $I^{n_{1}, \ldots, n_{m}}$ under which $\pi_{j}$ corresponds to $\pi_{j}^{n_{1}, \ldots, n_{m}}$. So the right-hand side of (3.4) equals:

$$
\sum_{\substack{m \geq 0 \\ n_{1}, \ldots, n_{m} \geq 1 \\ w \in n_{n_{1}, \ldots, n_{m}}}} \frac{1}{m ! n_{1} ! \ldots n_{m} !}\left|\left(A\left(\pi^{n_{1}, \ldots, n_{m}}\right) \times \prod_{j \in[m]} B\left(\pi_{j}^{n_{1}, \ldots, n_{m}}\right)\right)^{w \gamma}\right| x_{w} .
$$

Now any $w \in S_{n_{1}, \ldots, n_{m}}$ induces a permutation of the parts of $\pi^{n_{1}, \ldots, n_{m}}$, which corresponds to some $y \in S_{m}$ satisfying $n_{y(j)}=n_{j}$ for all $j \in[m]$. Write $\mathcal{C}(y)$ for its set of cycles, i.e. the orbits of $\langle y\rangle$ on $[m]$, and for $c \in \mathcal{C}(y)$, write $n_{c}$ for the common value of $n_{j}$ for $j \in c$. 
Clearly $w_{c}=w^{|c|}$ stabilizes each $\pi_{j}^{n_{1}, \ldots, n_{m}}$ for $j \in c$; choosing a particular representative of the cycle, we can identify $w_{c}$ with an element of $S_{n_{c}}$. It is easy to see that

$$
\left|\left(A\left(\pi^{n_{1}, \ldots, n_{m}}\right) \times \prod_{j \in[m]} B\left(\pi_{j}^{n_{1}, \ldots, n_{m}}\right)\right)^{w \gamma}\right|=\left|A[m]^{y \gamma}\right| \prod_{c \in \mathcal{C}(y)}\left|B\left[n_{c}\right]^{w_{c} \gamma^{|c|}}\right|,
$$

and

$$
x_{w}=\prod_{c \in \mathcal{C}(y)} x_{|c|} \circ x_{w_{c}} .
$$

Thus the term of (3.5) indexed by $w \in S_{n_{1}, \ldots, n_{m}}$ depends only on $y$ and the collection $\left\{w_{c}\right\}$. Since the number of $w$ giving rise to a fixed $y$ and $\left\{w_{c}\right\}$ is exactly $\frac{n_{1} ! \ldots n_{m} !}{\prod_{c \in \mathcal{C}(y)} n_{c} !}$, we can transform (3.5) into:

$$
\sum_{m \geq 0} \frac{1}{m !} \sum_{y \in S_{m}}\left|A[m]^{y \gamma}\right| \prod_{c \in \mathcal{C}(y)}\left(\sum_{n_{c} \geq 1} \frac{1}{n_{c} !} \sum_{w_{c} \in S_{n_{c}}}\left|B\left[n_{c}\right]^{w_{c} \gamma^{|c|}}\right| x_{|c|} \circ x_{w_{c}}\right) .
$$

That this equals $\left(Z_{A} \circ Z_{B}\right)(\gamma)$ is a matter of unravelling the definition of the latter.

An alternative proof of Theorem 3.1 will be sketched in the next section.

\section{Linear Analogues}

All the concepts mentioned so far have linear analogues. An $\mathbb{S}$-module, or tensor species in the terminology of [5], is a functor $U$ from $\mathbf{B}$ to the category Vect of vector spaces (over $\mathbb{C}$, say). In particular, $U[n]$ is a representation of $S_{n}$. Clearly any species $A$ can be linearized to give an $\mathbb{S}$-module $H^{0} A$, by composing with the functor $H^{0}: \mathbf{B} \rightarrow$ Vect defined by $H^{0}(J)=\{f: J \rightarrow \mathbb{C}\}$. (However, not every $\mathbb{S}$-module arises in this way.)

The characteristic of an $\mathbb{S}$-module $U$ is

$$
\operatorname{ch}(U):=\sum_{n \geq 0} \frac{1}{n !} \sum_{w \in S_{n}} \operatorname{tr}(w, U[n]) x_{w} \in \mathbb{Q}\left[\left[x_{1}, x_{2}, \ldots\right]\right] .
$$

This is the linearization of the definition of cycle index for species, in the sense that $\operatorname{ch}\left(H^{0} A\right)=Z_{A}$. (In the linear context it is customary to identify $\mathbb{Q}\left[\left[x_{1}, x_{2}, \ldots\right]\right]$ with a completion of the ring of symmetric functions, via the map sending $x_{i}$ to the $i$ th power sum.) Again we have specializations of $\operatorname{ch}(U)$ analogous to (2.2) and (2.3):

$$
\begin{aligned}
\operatorname{ch}(U)(x, 0,0, \ldots) & =\sum_{n \geq 0} \frac{\operatorname{dim} U[n]}{n !} x^{n}, \\
\operatorname{ch}(U)\left(x, x^{2}, x^{3}, \ldots\right) & =\sum_{n \geq 0} \operatorname{dim} U[n]^{S_{n}} x^{n} .
\end{aligned}
$$


The second equality follows from the linear analogue of (2.4):

$$
\operatorname{dim} U[n]^{S_{n}}=\frac{1}{n !} \sum_{w \in S_{n}} \operatorname{tr}(w, U[n]) .
$$

The definitions of addition, multiplication, and substitution for $\mathbb{S}$-modules are the linearizations of the definitions for species: that is, disjoint union is replaced by direct sum, and Cartesian product by tensor product. For instance, the definition of $U \circ V$ when $V(\emptyset)=0$ is:

$$
(U \circ V)(I):=\bigoplus_{\pi \in \operatorname{Par}(I)}\left(U(\pi) \otimes \bigotimes_{J \in \pi} V(J)\right) .
$$

Since $H^{0} A \circ H^{0} B$ is isomorphic to $H^{0}(A \circ B)$, the following is a generalization of Theorem 2.1:

Theorem 4.1 (Joyal) If $U$ and $V$ are $\mathbb{S}$-modules and $V(\emptyset)=0$, then

$$
\operatorname{ch}(U \circ V)=\operatorname{ch}(U) \circ \operatorname{ch}(V) .
$$

See [5, Chapitre 4]. As observed there, this amounts to an extension to analytic functors of the results on polynomial functors in [7, I, Appendix A].

In [3], Getzler generalizes the theory of $\mathbb{S}$-modules to the case of functors from $\mathbf{B}$ to a Karoubian ring category $\mathcal{R}$ over a field of characteristic 0 . A special case of his construction gives us the linear analogue of the $\Gamma$-species considered in the previous section: namely, we define an $(\mathbb{S} \times \Gamma)$-module to be a functor from $\mathbf{B}$ to the category of representations of $\Gamma$, over $\mathbb{C}$ say. The characteristic $\operatorname{ch}(U)$ of an $(\mathbb{S} \times \Gamma)$-module $U$ is the following element of $R_{\mathbb{Q}}(\Gamma)\left[\left[x_{1}, x_{2}, \ldots\right]\right]$, where $R_{\mathbb{Q}}(\Gamma)$ is the representation ring of $\Gamma$ with scalars extended to $\mathbb{Q}$ :

$$
\operatorname{ch}(U):=\sum_{n \geq 0} \sum_{E \in \widehat{\Gamma}} \frac{1}{n !} \sum_{w \in S_{n}} \operatorname{tr}\left(w, \operatorname{Hom}_{\Gamma}(E, U[n])\right)[E] x_{w} .
$$

Here $\widehat{\Gamma}$ is a set of representatives for the isomorphism classes of irreducible representations, and $\{[E] \mid E \in \widehat{\Gamma}\}$ is the correponding basis of $R_{\mathbb{Q}}(\Gamma)$.

Clearly any $\Gamma$-species $A$ gives rise to an $(\mathbb{S} \times \Gamma)$-module $H^{0} A$. If

$$
\chi: R_{\mathbb{Q}}(\Gamma)\left[\left[x_{1}, x_{2}, \ldots\right]\right] \rightarrow \mathbb{Q}\left[\left[x_{1}, x_{2}, \ldots\right]\right]_{\Gamma}
$$

is the linear map defined by $\chi([E])(\gamma)=\operatorname{tr}(\gamma, E)$ (an isomorphism onto the class functions on $\Gamma$ ), then

$$
\chi\left(\operatorname{ch}\left(H^{0} A\right)\right)=Z_{A} .
$$


This uses the following well-known identity for representations of $S_{n} \times \Gamma$ :

$$
\bigoplus_{E \in \widehat{\Gamma}} \operatorname{Hom}_{\Gamma}(E, V) \otimes E \cong V
$$

Plethysm in $R_{\mathbb{Q}}(\Gamma)\left[\left[x_{1}, x_{2}, \ldots\right]\right]$ can be defined using the $\lambda$-ring structure on $R_{\mathbb{Q}}(\Gamma)$ (see $[3$, Section 4]), or by the requirement $\chi(f \circ g)=\chi(f) \circ \chi(g)$. The analogue of Theorem 4.1 holds for $(\mathbb{S} \times \Gamma)$-modules also (the more general version for any Karoubian ring category is used implicitly in [3] but not stated). Thanks to (4.5), this provides another proof of Theorem 3.1.

\section{Species over $\mathbb{F}_{q}$}

Now we come to the main definition of this paper. Let $q$ be a prime power and write $\mathbb{F}_{q}$ for the finite field with $q$ elements. Fix an algebraic closure $\overline{\mathbb{F}_{q}}$, and let $\mathbb{F}_{q^{i}}$ be the unique subfield with $q^{i}$ elements, for $i \geq 1$. Let $\mathbb{F}_{q}$-var be the category of varieties over $\mathbb{F}_{q}$ and morphisms defined over $\mathbb{F}_{q}$. A species over $\mathbb{F}_{q}$ is a functor from $\mathbf{B}$ to $\mathbb{F}_{q}$-var.

If $X$ is a species over $\mathbb{F}_{q}$, then for all $n \geq 1, X[n]$ is a variety over $\mathbb{F}_{q}$, and as such it has a Frobenius endomorphism $F$. We will use the letter $F$ also for the induced permutation of the set $X[n]\left(\overline{\mathbb{F}_{q}}\right)$ of $\overline{\mathbb{F}_{q}}$-points, which satisfies

$$
X[n]\left(\overline{\mathbb{F}_{q}}\right)^{F^{i}}=X[n]\left(\mathbb{F}_{q^{i}}\right) .
$$

Also, $X[n]$ has an $S_{n}$-action defined over $\mathbb{F}_{q}$, which means that $X[n]\left(\overline{\mathbb{F}_{q}}\right)$ has an $S_{n}$-action commuting with $F$. It is well known that for any $w \in S_{n}$ and any $i \geq 1$,

$$
\left|X[n]\left(\overline{\mathbb{F}_{q}}\right)^{w F^{i}}\right|<\infty .
$$

(This is simply because $w F^{i}$ is the Frobenius endomorphism of a twisted $\mathbb{F}_{q^{i}}$-structure on $X[n]$.) We define the cycle index series of $X$ to be the sequence

$$
Z_{X}=\left(Z_{X}^{(1)}, Z_{X}^{(2)}, Z_{X}^{(3)}, \ldots\right)
$$

where

$$
Z_{X}^{(i)}:=\sum_{n \geq 0} \frac{1}{n !} \sum_{w \in S_{n}}\left|X[n]\left(\overline{\mathbb{F}_{q}}\right)^{w F^{i}}\right| x_{w} \in \mathbb{Q}\left[\left[x_{1}, x_{2}, \ldots\right]\right] .
$$

Since any finite set may be viewed as a variety over $\mathbb{F}_{q}$ with trivial Frobenius map, any species in the ordinary sense is a species over $\mathbb{F}_{q}$ (with a cycle index series in which every term is the cycle index series in the ordinary sense). We will see some less trivial examples of species over $\mathbb{F}_{q}$ in the next section.

Note that $\left|X[n]\left(\overline{\mathbb{F}_{q}}\right)^{w F^{i}}\right|$ depends only on the conjugacy class of $w$, so as in the case of ordinary species, all the numbers $\left|X[n]\left(\overline{\mathbb{F}_{q}}\right)^{w F^{i}}\right|$ can be recovered from $Z_{X}^{(i)}$. Again, we have 
two significant specializations:

$$
\begin{aligned}
Z_{X}^{(i)}(x, 0,0, \ldots) & =\sum_{n \geq 0} \frac{\left|X[n]\left(\mathbb{F}_{q^{i}}\right)\right|}{n !} x^{n}, \\
Z_{X}^{(i)}\left(x, x^{2}, x^{3}, \ldots\right) & =\sum_{n \geq 0}\left|\left(S_{n} \backslash X[n]\right)\left(\mathbb{F}_{q^{i}}\right)\right| x^{n} .
\end{aligned}
$$

To get this latter equation, we have used

$$
\left|\left(S_{n} \backslash X[n]\right)\left(\mathbb{F}_{q^{i}}\right)\right|=\frac{1}{n !} \sum_{w \in S_{n}}\left|X[n]\left(\overline{\mathbb{F}_{q}}\right)^{w F^{i}}\right| .
$$

This is the special case $A[n]=X[n]\left(\overline{\mathbb{F}_{q}}\right), \gamma=F^{i}$ of (3.3), which in fact does not require $|A[n]|$ itself to be finite, as long as all $\left|A[n]^{w \gamma}\right|$ are.

It is clear that the definitions of addition and multiplication for ordinary species make sense for species over $\mathbb{F}_{q}$, and correspond to the operations of addition and multiplication on cycle index series, defined on each term of the sequence independently. The definition of substitution also makes perfect sense in this new context. The correct definition of plethysm of two sequences of elements of $\mathbb{Q}\left[\left[x_{1}, x_{2}, \ldots\right]\right]$ is readily guessed: if $f=\left(f^{(1)}, f^{(2)}, \ldots\right)$ and $g=\left(g^{(1)}, g^{(2)}, \ldots\right)$, where all $g^{(i)}$ have zero constant term, then

$$
(f \circ g)^{(i)}:=f^{(i)}\left(g^{(i)}\left(x_{1}, x_{2}, \ldots\right), g^{(2 i)}\left(x_{2}, x_{4}, \ldots\right), \ldots\right) .
$$

With these definitions, the obvious extension of Theorem 2.1 is true:

Theorem 5.1 If $X$ and $Y$ are two species over $\mathbb{F}_{q}$ with $Y(\emptyset)=\emptyset$,

$$
Z_{X \circ Y}=Z_{X} \circ Z_{Y}
$$

Proof: This can be proved in exactly the same way as Theorem 3.1; the condition (5.1) is the only finiteness required. Alternatively, we can make our sets finite by brute force: to any species $X$ over $\mathbb{F}_{q}$ we can associate a $\mathbb{Z}$-species $X^{\text {fin }}$ defined by

$$
X^{\text {fin }}(I)=X(I)\left(\mathbb{F}_{q^{\mid l ! !}}\right),
$$

with $\mathbb{Z}$ acting by the powers of $F$. It is clear that

$$
X[n]\left(\overline{\mathbb{F}_{q}}\right)^{w F^{i}}=X^{\mathrm{fin}}[n]^{w F^{i}},
$$

whence $Z_{X}^{(i)}=Z_{X^{\text {fn }}}(i)$. So in fact we can deduce Theorem 5.1 from Theorem 3.1, which, as we have seen, may be proved either by direct computation or by linearizing.

To remove any last vestige of surprise at Theorem 5.1, let us sketch a different linear approach to proving it. The Frobenius map $F$ induces an endomorphism $F^{*}$ of the $l$-adic 
cohomology groups $H_{c}^{j}\left(X[n], \overline{\mathbb{Q}_{l}}\right)$, commuting with the $S_{n}$-action. By the Grothendieck Trace Formula,

$$
Z_{X}^{(i)}=\sum_{n \geq 0} \frac{1}{n !} \sum_{\substack{w \in S_{n} \\ j \in \mathbb{Z}}}(-1)^{j} \operatorname{tr}\left(w\left(F^{*}\right)^{i}, H_{c}^{j}\left(X[n], \overline{\mathbb{Q}_{l}}\right)\right) x_{w} .
$$

In the terminology of [3, Section 5], the right-hand side is the "associated Euler characteristic" of the "Künneth functor" $H_{c}^{\bullet}\left(X, \overline{\mathbb{Q}_{l}}\right)$ with values in the Karoubian ring category of $\overline{\mathbb{Q}_{l}}$-vector spaces with an endomorphism. So Theorem 5.1 follows from the generalization of Theorem 4.1 that is used implicitly in [3]. However, note that Getzler's favoured special case, where the linear category is that of mixed Hodge structures, does not quite imply Theorem 5.1, since it gives a coarser Grothendieck group where eigenvalues of $F^{*}$ with the same absolute value are not distinguished.

\section{Configuration spaces}

Let $X$ be a fixed irreducible nonsingular variety over $\mathbb{F}_{q}$. We will write $X$ also for the species over $\mathbb{F}_{q}$ defined by

$$
X(I)= \begin{cases}X, & \text { if }|I|=1 \\ \emptyset, & \text { otherwise }\end{cases}
$$

The cycle index series of this species is given by

$$
Z_{X}^{(i)}=\left|X\left(\mathbb{F}_{q^{i}}\right)\right| x_{1} .
$$

Imitating the notation of [3], we define three further species over $\mathbb{F}_{q}$ which depend on $X$ :

(1) The first is the species $T_{X}$ of tuples of points in $X$, defined by $T_{X}(I)=X^{I}$. For instance, $T_{X}[n]\left(\overline{\mathbb{F}_{q}}\right)$ is the set of $n$-tuples $\left(p_{1}, \ldots, p_{n}\right)$ of points in $X\left(\overline{\mathbb{F}_{q}}\right)$.

(2) The second is the species $F_{X}$ of tuples of distinct points in $X$, defined by setting $F_{X}(I)$ to be the complement of the diagonals in $T_{X}(I)$. For instance, $F_{X}[n]\left(\overline{\mathbb{F}_{q}}\right)$ is the configuration space consisting of all $n$-tuples $\left(p_{1}, \ldots, p_{n}\right)$ as above where $p_{i} \neq p_{j}$ for all $i \neq j$.

(3) The third is the species $F M_{X}$ defined by setting $F M_{X}(I)$ to be the Fulton-MacPherson "compactification" of $F_{X}(I)$, as defined in [2]. (The word "compactification" only applies when $X$ is a compact complex variety; but their construction works for any irreducible nonsingular variety over any ground field.)

In this section we will consider the problem of computing the cycle index series of these species over $\mathbb{F}_{q}$, in terms of the numbers $\left|X\left(\mathbb{F}_{q^{i}}\right)\right|$.

We need the following obvious principle: 
Lemma 6.1 Let $Y$ be a species over $\mathbb{F}_{q}$. Suppose that:

(1) Each variety $Y(I)$ has a finite stratification

$$
Y(I)=\bigcup_{\alpha \in A_{I}} Y(I)_{\alpha}
$$

into locally closed subvarieties defined over $\mathbb{F}_{q}$.

(2) For any bijection $f: I \stackrel{\sim}{\rightarrow} I^{\prime}$,

$$
\begin{gathered}
Y(f)\left(Y(I)_{\alpha}\right)=Y\left(I^{\prime}\right)_{\widehat{f}(\alpha)}, \\
\text { for some bijection } \widehat{f}: A_{I} \stackrel{\sim}{\rightarrow} A_{I^{\prime}} .
\end{gathered}
$$

Define a species $\widehat{Y}$ over $\mathbb{F}_{q}$ by “cutting” $Y$ up into the pieces of this stratification; in other words,

$$
\widehat{Y}(I):=\coprod_{\alpha \in A_{I}} Y(I)_{\alpha}
$$

Then $Z_{\widehat{Y}}=Z_{Y}$.

Proof: The distinction between $Y$ and $\widehat{Y}$ is solely that the pieces $Y(I)_{\alpha}$ which are "glued together" in $Y(I)$ are disconnected in $\widehat{Y}(I)$; this does not affect the number of Frobenius fixed points. (Indeed, if we pass to the truncated finite versions as in the proof of Theorem 5.1, we get an isomorphism $\widehat{Y}^{\text {fin }} \cong Y^{\text {fin }}$ of $\mathbb{Z}$-species.)

Of course, the first of the three species presents no difficulty at all.

Lemma 6.2 For $i \geq 1$,

$$
Z_{T_{X}}^{(i)}=\exp \left(\sum_{n \geq 1} \frac{\left|X\left(\mathbb{F}_{q^{i n}}\right)\right| x_{n}}{n}\right) .
$$

Proof: This is easy to prove directly from the definition. A slightly cleaner proof is the following: if $E$ denotes the species of sets, defined by $E(I)=\{I\}$ for all $I$, then we have an obvious isomorphism

$$
E \circ X \cong T_{X}
$$

So by Theorem 5.1, $Z_{T_{X}}=Z_{E} \circ Z_{X}$. Here $Z_{E}$ means the constant sequence each of whose terms is $Z_{E}$ in the sense of ordinary species. It is almost trivial to show that

$$
Z_{E}=\exp \left(\sum_{n \geq 1} \frac{x_{n}}{n}\right),
$$

and combining this with (6.1) gives the result. 
The second is not much harder.

Lemma 6.3 For $i \geq 1$,

$$
Z_{F_{X}}^{(i)}=\exp \left(\sum_{n \geq 1} \frac{\left|X\left(\mathbb{F}_{q^{i n}}\right)\right|}{n} \sum_{m \geq 1} \frac{\mu(m)}{m} \log \left(1+x_{n m}\right)\right) .
$$

Proof: Again, this can be proved by directly counting Frobenius fixed points: it is equivalent to [6, Lemma 4.2]. Another approach is to use Theorem 5.1 in the following way (compare the proof of [3, Theorem 5.6]). The variety $T_{X}(I)$ of $I$-tuples is stratified according to which components of the tuple are equal. Let $\widehat{T}_{X}$ be the species over $\mathbb{F}_{q}$ obtained by cutting up the varieties $T_{X}(I)$ into these pieces, as in Lemma 6.1. Then clearly

$$
\widehat{T}_{X} \cong F_{X} \circ E_{+},
$$

where $E_{+}$is the species defined by

$$
E_{+}(I)= \begin{cases}\{I\}, & \text { if }|I| \geq 1 \\ \emptyset, & \text { if } I=\emptyset .\end{cases}
$$

Hence

$$
Z_{T_{X}}=Z_{\widehat{T}_{X}}=Z_{F_{X}} \circ Z_{E_{+}} \cdot
$$

Now the plethystic inverse of

$$
Z_{E_{+}}=Z_{E}-1=\exp \left(\sum_{n \geq 1} \frac{x_{n}}{n}\right)-1
$$

is well known to be

$$
Z_{E_{+}}^{\langle-1\rangle}:=\sum_{m \geq 1} \frac{\mu(m)}{m} \log \left(1+x_{m}\right) .
$$

(This can also be interpreted as the cycle index series of the virtual species $E_{+}^{\langle-1\rangle}-$ see [1, Section 2.5].) Multiplying (in the plethystic sense) both sides of (6.3) on the right by $Z_{E_{+}}^{\langle-1\rangle}$ and using Lemma 6.2, we get the result.

For the third of the species, the Fulton-MacPherson "compactifications", a result such as Theorem 5.1 is probably essential. In [3, Section 6], Getzler treats the analogous problem for the case of complex varieties, using the variant of $\mathbb{S}$-modules where the linear category is that of mixed Hodge structures. (This is an equivariant generalization of the work done in $[8$, Section 2].) As we will see, his argument works just as well in the context of species 
over $\mathbb{F}_{q}$. This is not surprising: changing the base field from $\mathbb{C}$ to $\overline{\mathbb{F}_{q}}$ and using $l$-adic instead of singular cohomology accounts for most of the difference between the two contexts, and the remainder is the minor change from mixed Hodge structures, which encode only the absolute value of the Frobenius eigenvalues, to our cycle index series, which encode the eigenvalues themselves (see the remark at the end of the previous section). But some readers may prefer the following argument, which avoids using cohomology.

The construction of $F M_{X}[n]$ in [2] involves blowing up the union of the diagonals in $X^{n}$ in a certain way. Intuitively speaking, when the components of an $n$-tuple in $F_{X}[n]$ vary in such a way that some of them become equal, the limit point in $F M_{X}[n]$ records not just the final value of the $n$-tuple in $X^{n}$, but some extra data (the screens) encoding the relative rates of coincidence. The main point of Getzler's argument is that the subvariety parametrizing the extra data depends only on the dimension of $X$ and which components are equal, not the components themselves. Hence, as with $T_{X}$, we can cut up $F M_{X}$ according to which components are equal, and the resulting species $\widehat{F M}_{X}$ will be isomorphic to $F_{X} \circ P_{\operatorname{dim} X}$ for some family $\left\{P_{k} \mid k \geq 0\right\}$ of species over $\mathbb{F}_{q}$ (defined below). Thus

$$
Z_{F M_{X}}=Z_{\widehat{F M}_{X}}=Z_{F_{X}} \circ Z_{P_{\operatorname{dim} X}} .
$$

(This is the analogue of [3, Proposition 6.9].) Since we know $Z_{F_{X}}$ by Lemma 6.3, we need only compute $Z_{P_{k}}$ for all $k \geq 0$. In fact, a recursive procedure for calculating the terms of $Z_{P_{k}}^{(i)}$ is the best we can do.

The species $P_{k}$ is defined by setting $P_{k}(I)$ to be the variety of screens based on $\mathbb{A}^{k}$ with marked points labelled by $I$. (By convention, this is empty if $I$ is empty, and a single point if $|I|=1$.) This variety has an open subvariety $Q_{k}(I)$ defined by

$$
Q_{k}(I)= \begin{cases}\left(\mathbb{G}_{a}^{k} \rtimes \mathbb{G}_{m}\right) \backslash F_{\mathbb{A}^{k}}(I), & \text { if }|I| \geq 2 \\ \emptyset, & \text { otherwise. }\end{cases}
$$

The quotient here is by the simultaneous action of the affine transformation group $\mathbb{G}_{a}^{k} \rtimes \mathbb{G}_{m}$ on all components of the $I$-tuple. This is well defined in $\mathbb{F}_{q}$-var; indeed the group action is free (unless $k=0$, when $Q_{0}(I)=\emptyset$ for all $I$ ). For example, if $|I|=2, Q_{k}(I) \cong \mathbb{P}^{k-1}$.

It is clear that $Q_{k}$ is a species over $\mathbb{F}_{q}$. We have the following analogue of [3, Proposition 6.6]:

Lemma 6.4 For $i \geq 1$,

$$
Z_{Q_{k}}^{(i)}=\frac{\exp \left(\sum_{m, n \geq 1} \frac{\mu(m) q^{i n k}}{m n} \log \left(1+x_{m n}\right)\right)-1-q^{i k} x_{1}}{q^{i k}\left(q^{i}-1\right)} .
$$

Proof: It is a familiar fact, following from Lang's Theorem, that if $Z$ is a variety over $\mathbb{F}_{q}$ with a free action of the connected algebraic group $G$, also defined over $\mathbb{F}_{q}$, then the obvious map

$$
G\left(\mathbb{F}_{q}\right) \backslash Z\left(\mathbb{F}_{q}\right) \rightarrow(G \backslash Z)\left(\mathbb{F}_{q}\right)
$$


is a bijection. An easy variant is that if $Z$ also has an action of the finite group $W$, defined over $\mathbb{F}_{q}$ and commuting with the action of $G$, then for all $w \in W$,

$$
G\left(\mathbb{F}_{q}\right) \backslash Z\left(\overline{\mathbb{F}_{q}}\right)^{w F} \rightarrow(G \backslash Z)\left(\overline{\mathbb{F}_{q}}\right)^{w F}
$$

is a bijection. Applying this to the case where $Z=F_{\mathbb{A}^{k}}[n], G=\mathbb{G}_{a}^{k} \rtimes \mathbb{G}_{m}$, and $W=S_{n}$, with $q$ replaced by $q^{i}$, we get

$$
Z_{Q_{k}}^{(i)}=\frac{Z_{F_{\mathbb{A}^{k}}}^{(i)}-1-q^{i k} x_{1}}{q^{i k}\left(q^{i}-1\right)} .
$$

Using Lemma 6.3, we are done.

The open subvariety $Q_{k}(I)$ is just one stratum of a stratification of the variety $P_{k}(I)$. The strata are parametrized by the set $\mathcal{T}(I)$ of isomorphism classes of rooted trees, with a bijection between $I$ and the set of leaves, in which every internal vertex has indegree $\geq 2$. Here we are following the terminology of [1, Section 3.1]: the indegree of a vertex is the cardinality of its fibre, i.e. the set of edges incident with the vertex which lead away from the root. The leaves are the vertices of indegree 0 , and the internal vertices are the rest. (In the terminology of [2], $\mathcal{T}(I)$ is the set of nests of subsets of $I$ which include $I$ itself.) Note that $\mathcal{T}(\emptyset)=\emptyset$, and $|I|=1 \Rightarrow|\mathcal{T}(I)|=1$.

By abuse of notation, we write $\mathcal{T}(I)$ also for a set of representatives of the isomorphism classes of trees as above. If $T \in \mathcal{T}(I)$, define

$$
Q_{k}(T):=\prod_{v \in \operatorname{Int}(T)} Q_{k}(\operatorname{Fibre}(v)),
$$

where $\operatorname{Int}(T)$ denotes the set of internal vertices of $T$ and Fibre $(v)$ denotes the fibre of $v$. This is precisely the stratum of $P_{k}(I)$ corresponding to $T$. (The open stratum $Q_{k}(I)$ corresponds to the tree in which the root is the sole internal vertex.)

Cutting up $P_{k}(I)$ into these strata as in Lemma 6.1, we obtain the species $\widehat{P}_{k}$, defined by

$$
\widehat{P}_{k}(I)=\coprod_{T \in \mathcal{T}(I)} Q_{k}(T)
$$

Extending the definition of $\left[1\right.$, Section 4.1] to the case of species over $\mathbb{F}_{q}$, we have a twosort species $B_{1+Q_{k}}$ of $\left(1+Q_{k}\right)$-enriched rooted trees with internal vertices of one sort and leaves of another sort. To obtain $\widehat{P}_{k}$ from $B_{1+Q_{k}}$, we take isomorphism types according to the internal vertex sort, as in [1, Section 2.4]. Hence [1, (4.1.34)] implies the following isomorphism of species over $\mathbb{F}_{q}$ :

$$
\widehat{P}_{k} \cong E_{1}+Q_{k} \circ \widehat{P}_{k}
$$

(The morphism from the varieties on the left to those on the right works by "splitting the tree at the root", to create an assemblage of smaller trees. This result is the analogue of [3, Theorem 6.4].) We can thus deduce: 
Proposition 6.5 For all $k \geq 0$,

$$
Z_{P_{k}}=x_{1}+Z_{Q_{k}} \circ Z_{P_{k}}
$$

Proof: This follows from Theorem 5.1, (6.5), and Lemma 6.1.

Now in the grading on $\mathbb{Q}\left[\left[x_{1}, x_{2}, \ldots\right]\right]$ defined by $\operatorname{deg} x_{n}=n$, all the series $Z_{Q_{k}}^{(i)}$ start in degree 2. So Proposition 6.5 expresses the degree $n$ term of $Z_{P_{k}}^{(i)}$ as a function of the coefficients of $Z_{Q_{k}}^{(i)}$ and terms of various $Z_{P_{k}}^{(j)}$ of degree less than $n$. Since we know $Z_{Q_{k}}$ by Lemma 6.4, this gives our desired recursion. Once we have computed all $Z_{P_{k}}^{(j)}$ up to terms of degree $n$, (6.4) gives a formula for the terms of $Z_{F M_{X}}^{(i)}$ of degree $\leq n$ in terms of the coefficients of $Z_{F_{X}}^{(i)}$, which we know by Lemma 6.3.

\section{Acknowledgments}

This work was supported by Australian Research Council grant DP0344185.

\section{References}

1. F. Bergeron, G. Labelle, and P. Leroux, Combinatorial Species and Tree-like Structures, vol. 67 of Encyclopedia of Mathematics and its Applications, Cambridge University Press, 1998.

2. W. Fulton and R. Macpherson, "A compactification of configuration spaces," Ann. of Math. 139 (1994), 183225.

3. E. Getzler, "Mixed Hodge structures of configuration spaces," preprint, MPI-96-61, http://arxiv.org/abs/alggeom/9510018.

4. A. Joyal, "Une théorie combinatoire des séries formelles," Adv. Math. 42 (1981), 1-82.

5. A. Joyal, "Foncteurs analytiques et espèces de structures," in Combinatoire Énumérative (Quebec, 1985), vol. 1234 of Lecture Notes in Mathematics, Springer, Berlin, 1986, pp. 126-159.

6. M. Kisin and G.I. Lehrer, "Equivariant Poincaré polynomials and counting points over finite fields," J. Algebra 247 (2002), 435-451.

7. I.G. Macdonald, Symmetric Functions and Hall Polynomials, 2nd ed., Oxford Univ. Press, 1995.

8. Y.I. Manin, "Generating functions in algebraic geometry and sums over trees," in The Moduli space of Curves (Texel Island, 1994), vol. 129 of Progress in Mathematics, Birkhauser Boston, Boston, 1995, pp. 401-417.

9. Y.I. Manin, Frobenius Manifolds, Quantum Cohomology, and Moduli Spaces, vol. 47 of American Mathematical Society Colloquium Publications, American Mathematical Society, 1999. 
